\title{
Feature
}

\section{Claudication in the Young Patient}

RICHARD T. KRUG, M.D., KEITH D. CALLIGARO, M.D., and MATTHEW J. DOUGHERTY, M.D.

ABSTRACT In young patients, generally defined as those under 40 years of age, intermittent claudication (IC) is relatively rare and represents a different spectrum of pathology from IC in older patients. Atherosclerosis in older patients is the most common etiology, but appears to represent a distinct clinical entity with a more virulent course. Furthermore, other causes of lower extremity ischemia play a more prominent role in young patients. These include: (1) popliteal artery entrapment syndrome; (2) traumatic occlusion; (3) adventitial cystic disease; (4) exercise induced compartment syndrome; (5) repetitive trauma; (6) congenital arterial anomalies; and (7) ergotism. We outline the important features of the various causes of (IC) in the young and review the facets of diagnosis and management of these patients.

Keywords Intermittent claudication, young patients, etiology, management

Claudication from vascular insufficiency is an unusual problem in patients under 40 years of age. Young patients make up between 1.4 and $7 \%$ of all those treated for lower extremity ischemia. A population-based study indicates an incidence of 14.6/100,000 person years for patients under $40 .{ }^{1}$ Because the diagnosis is relatively unusual in this age group, its recognition is frequently delayed. Symptoms tend to be attributed to musculoskeletal or other causes, postponing the correct diagnosis and treatment. ${ }^{2}$ An awareness of this problem and its unique diagnostic and therapeutic challenges in young, active patients is crucial in avoiding the extended morbidity associated with this delay. The dif-

R.T.K., Fellow, Section of Vascular Surgery, Pennsylvania Hospital; K.D.C., Chief, Section of Vascular Surgery, Pennsylvania Hospital; Associate Clinical Professor, University of Pennsylvania School of Medicine, Philadelphia; M.J.D., Section of Vascular Surgery, Pennsylvania Hospital; Assistant Clinical Professor, University of Pennsylvania School of Medicine, Philadelphia, PA.

Copyright (C) 2000 by Thieme Medical Publishers, Inc., 333 Seventh Avenue, New York, NY 10001, USA.

Tel.: +1(212) 584-4662. 0894-8046,p;2000,13,2,27,38,ftx,en; pvs000099 
ferential diagnosis in young patients with claudication includes atherosclerotic occlusive disease as well as a spectrum of other disorders responsible for arterial insufficiency of the lower extremities: (1) popliteal artery entrapment syndrome; (2) traumatic occlusion; (3) adventitial cystic disease; (4) exercise induced compartment syndrome; (5) repetitive trauma; (6) congenital arterial anomalies, and (7) ergotism. We will review the diagnosis and management of this unusual problem, including the important facets of each etiology.

\section{PREMATURE ATHEROSCLEROSIS}

As in the elderly, the most common cause of lower extremity ischemia in patients under age forty is atherosclerosis. ${ }^{2}$ There are, however, important differences in the clinical behavior of atherosclerosis in young patients. Differences in demographics, risk factors, anatomic distribution, and prognosis suggest that atherosclerosis in the young should be regarded as a distinct clinical entity, rather than simply early onset of the same disease.

Claudication in the elderly, while a marker for systemic atherosclerosis, has a relatively benign course with respect to ischemic complications in the lower extremities. $^{3}$ In most series, fewer than $10 \%$ of patients who present with lower extremity claudication ultimately require amputation. ${ }^{4}$ By contrast, young patients with ischemic symptoms in the lower extremities appear to have a much more virulent form of disease. ${ }^{5}$ In the study by Levy et al. of 109 patients under 40 years of age with lower extremity ischemia from atherosclerosis, $90 \%$ required either revascularization or amputation within 3 years of onset of symptoms. Furthermore, $27 \%$ of patients overall and $50 \%$ of those who initially presented with a threatened limb went on to major amputation during the 6-year study period. ${ }^{1}$ Bouhoutsos and Martin demonstrated a strong negative impact of young age on rates of both graft patency and limb salvage. ${ }^{6}$ At six year follow-up after femoropopliteal reconstruction, amputations had been performed on $71 \%$ of patients aged 33 to $40,35 \%$ of those aged 41 to 50 , and $14 \%$ of those over age 50 .

The anatomic distribution of atherosclerosis in young patients is another distinguishing feature. When patients of all ages with claudication are considered, infrainguinal disease predominates. ${ }^{7}$ By contrast, most studies of young patients with claudication demonstrate a preponderance of aortoiliac disease. ${ }^{1,2,5,7,8}$ Furthermore, the pattern of disease tends to be segmental rather than the diffuse disease seen in older patients. ${ }^{8}$

Further distinguishing the younger age group is a higher prevalence of women, compared to claudicants at large. In DeBakey's series of patients under $40,38 \%$ were female, similar to the $34 \%$ rate in Levy's series. ${ }^{8}, 9$ Fully half of patients under age 30 in each series were women. These findings are in sharp contrast to only $14 \%$ women in DeBakey's total series of 3500 patients.

The primary atherosclerotic risk factor in young patients, as in the general population, is cigarette smoking. In most reports, $90 \%$ or more of patients 
under age 40 with claudication caused by atherosclerosis were smokers. 5,7,9 Atypical risk factors, however, assume a greater role in the disease process of young patients. Hypercoagulable states were discovered in $15 \%$ of young patients with lower extremity arterial occlusive disease when studied retrospectively, and prospective studies identify far more patients. Using a wide range of laboratory tests of hypercoagulability, 76 to $90 \%$ of patients have been found to have abnormalities. These disorders of coagulation correlate with higher rates of graft failure and of amputation.1,9,10

Another risk factor prevalent in younger patients, and particularly in women, is hypoplasia of the aortoiliac system. ${ }^{11}$ Diffuse narrowing of the aortoiliac segment, and often an "hourglass stenosis" may represent congenital hypoplasia which, when combined with typical atherosclerosis, can lead to the early onset of claudication. This may be a contributing factor in $15 \%$ of young patients. ${ }^{1}$ Other factors such as homocystinuria, which can cause early atherosclerosis, should also be considered. Finally, Crohn's colitis has been reported to have an association with lower extremity arterial occlusions from premature atherosclerosis. ${ }^{12}$ A chronic inflammatory state and disordered coagulation in patients with Crohn's may contribute to this association.

Treating these patients presents a particular challenge. Because they are young, active, and generally still in the work force, their claudication is typically a more severe liability than in older patients. For this reason, some authors have advocated aggressive intervention based on acceptable results in the short term. ${ }^{7,8}$ On the other hand, the results of intervention have been shown after long term follow-up to be substantially worse than in older patients, with failure rates on the order of $70 \% .6,13$

In general, intervention should be reserved for those patients with the most disabling symptoms or with severe, limb threatening ischemia. For those young patients who do require intervention, an aortoiliac distribution of disease is more common than in the elderly, and aortobifemoral bypass offers the best long-term outcome. When infrainguinal bypass is required, every effort should be made to use autologous vein as the conduit, as the long-term patency is clearly superior to that of prosthetic grafts. The management of these patients should include aggressive efforts at smoking cessation, both to slow progression of disease, and to improve the outcome of intervention. Patients under age 40 who require arterial reconstruction should be investigated for hypercoagulable states, as a significant number may require anticoagulation to prevent graft failure.

\section{POPLITEAL ARTERY ENTRAPMENT SYNDROME}

The most commonly reported cause of claudication in young patients other than atherosclerosis is popliteal artery entrapment syndrome, in which the popliteal artery is compressed by muscle or by a fibrous band. The incidence of the anatomic defect was $3.5 \%$ in a series of 86 anatomic cadaver dissec- 
tions. ${ }^{14}$ The typical patient is an otherwise healthy, athletic man with calf pain induced by strenuous exercise. Males predominate by a ratio of 9:1 in this disorder. The presentation is that of intermittent claudication in about $70 \%$ of cases. A further $25 \%$ present with paresthesias or critical ischemia, and the remainder with unusual symptoms, including digital ulceration caused by embolization from a post-stenotic aneurysm. ${ }^{15}$ The time course can be one of gradual onset, or of sudden onset during an episode of strenuous exercise, the latter being suggestive of occlusion of a compressed artery.

The etiology of the disorder is a defect in embryological development. The popliteal artery develops from the union of two precursors- the poplitea profunda artery and the superficialis poplitea. The major blood supply of the leg is initially via the profunda which runs between the popliteus muscle and the tibia. During development the majority of flow is transferred to the superficialis, which runs posterior to the popliteus. At about the same time, the medial head of the gastrocnemius muscle migrates cephalad to attach to the medial epicondyle of the femur. A disruption of this process will lead to abnormal anatomic relationships among these structures. ${ }^{14}$

The variations in anatomy that result from this disordered development form the basis of the classification scheme proposed by Rich et al. in which five types of popliteal entrapment are described ${ }^{16}$ (Fig. 1). In type 1, the popliteal artery takes an abnormal course medial to the medial head of the gastrocnemius muscle. In type 2 , the position of the artery is relatively normal, but the medial head of the gastrocnemius inserts lateral to the artery. In type 3, a slip of the medial head of the gastrocnemius inserts laterally. In type 4 the compression is caused by the popliteus muscle or by a fibrous band overlying it. Type 5 represents any of types 1 through 4 with the additional involvement of the popliteal vein. Recently, a "type 6" has been proposed in which there is no clear anatomic abnormality. This variation has been associated with over-training, and is designated "functional" popliteal entrapment syndrome. ${ }^{17}$

The chronic compression of the popliteal artery in these conditions eventually leads to injury of the vessel and pathologic changes. These have been classified as stage I, in which adventitial thickening and fibrosis occurs, stage II in which fibrous replacement extends into the media, and stage III in which involvement of the intima predisposes the artery to thrombosis. In over half of patients, the popliteal artery is thrombosed at the time of presentation, and because of the pathologic changes described, thrombectomy is not successful. These cases require surgical replacement of the diseased segment. ${ }^{18}$

The diagnosis of popliteal artery entrapment syndrome is suggested by the clinical setting of a young, healthy patient with intermittent claudication. Physical exam may demonstrate diminished pedal pulses at rest, or during active plantar flexion or passive dorsiflexion of the foot. Duplex ultrasound has been used to help confirm abnormal compression of the popliteal artery during these maneuvers. ${ }^{19}$ It is important to interpret these results in the 

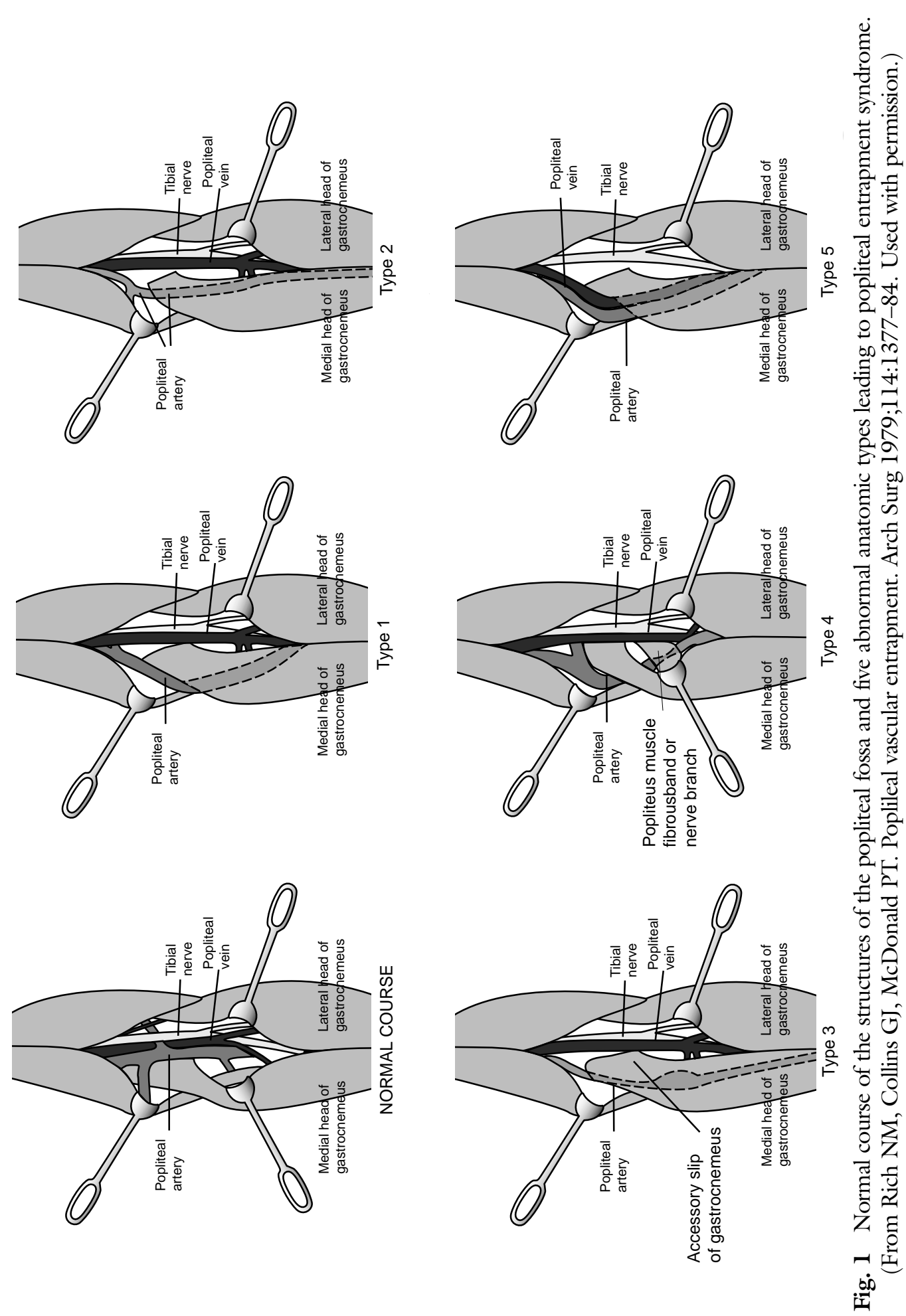
context of the clinical setting, as more than half of normal subjects in one study were able to occlude their popliteal arteries with positional changes. ${ }^{20}$

Angiography remains the gold standard for diagnosis. Findings at rest include medial deviation of the proximal popliteal artery, occlusion of the mid artery, or post-stenotic dilatation. If these findings are absent at rest, films should be repeated during either active plantar flexion or passive dorsiflexion. Popliteal entrapment will be demonstrated by medial deviation or by compression. ${ }^{18}$ Both legs should be studied, as about $20 \%$ of cases are bilateral. ${ }^{21}$

Treatment of this syndrome is surgical. In patients who are diagnosed early, there may be little or no irreversible arterial injury, in which case only a release of the compressing structure is necessary. On the other hand, if there is evidence of stenosis, aneurysm or occlusion, then interposition grafting or bypass is required. The surgical approach can be either posterior or medial. The posterior approach may afford better delineation of the anatomic defect, and is probably preferred in cases in which no arterial reconstruction or only a short interposition graft is needed. On the other hand, if the popliteal artery is occluded to the level of the trifurcation, or over a long segment, a medial approach should be used in order to gain adequate proximal and distal control. ${ }^{18}$

Patients with "functional" popliteal entrapment and no obvious anatomic lesion have been treated successfully by lysis of the medial head of the gastrocnemius muscle through a medial incision. ${ }^{18}$ Turnipseed has advocated treatment of these patients by release of the soleus muscle from the tibia and resection of the plantaris muscle. ${ }^{17}$

All angiographically confirmed cases of popliteal entrapment should be corrected. This includes asymptomatic cases discovered incidentally or on investigation of the asymptomatic contralateral limb of a patient with popliteal entrapment. The morbidity of early operation is low, and the consequences of allowing a lesion to progress to thrombosis in a young, active patient are potentially grave. ${ }^{15}$

\section{TRAUMATIC OCCLUSION}

Delayed presentation of traumatic arterial occlusion deserves mention, and in one series was the second most common cause of claudication in young patients after atherosclerosis. ${ }^{2}$ In this series two patients suffered arterial trauma in infancy but did not develop symptoms until adulthood. Two others were injured in motorcycle accidents and suffered from claudication afterward, but were not referred for evaluation for up to 1 year. All four patients underwent successful arterial reconstruction.

\section{ADVENTITIAL CYSTIC DISEASE}

Adventitial cystic disease is a rare cause of claudication in which fluid-filled cysts form in the arterial wall, resulting in stenosis or occlusion. A review of 
the world literature revealed a total of 323 reported cases since the first description in 1947. The popliteal artery is by far the most commonly reported site of involvement (82\%) with the iliofemoral segment a distant second $(9 \%)$. There are occasional reports of lesions in the radial and ulnar arteries, as well as in venous sites. ${ }^{22}$ About $80 \%$ of patients are male, and typically present around age 40 , although patients ranging in age from 11 to 70 have been reported. ${ }^{23}$

The clinical presentation is typically with a sudden onset of unilateral claudication that is generally severe. Because of the severity, patients generally present after a relatively short time. Physical examination may reveal a bruit in the popliteal fossa and loss of pulses with sharp flexion of the knee. If the lesion has progressed to occlusion, as is seen in one quarter of cases, ${ }^{23}$ popliteal and pedal pulses will be absent at rest.

The etiology of this lesion remains uncertain. One theory, however, appears to be gaining more widespread support. Several authors have proposed that this condition is the result of a defect in embryological development. The pathologic cysts may arise from cell rests derived from the condensing mesenchyme of adjacent developing joints. Evidence to support this hypothesis includes the fact that all reported cases occur in nonaxial arteries that form in proximity to joints at the embryologic stage when this condensation occurs. Importantly, those vessels that arise from the embryologic axial arteries, which form prior to this process, are not involved in any reported cases of adventitial cystic disease. Since the composition of the fluid within cysts differs from that of synovial fluid, it is presumed that these cells rests are not synovial cells, but less differentiated mesenchymal cells. ${ }^{22}$

Other potential etiologies have been proposed. These cysts could simply represent ectopic ganglion cysts. In that case, however, the cyst fluid would be expected to match synovial fluid in composition, which it does not. ${ }^{22}$ One popular theory has been that repetitive trauma causes cystic degeneration of the artery. If this were the case, however, one would expect to see this lesion in association with over-training, or in high-level competition athletes, neither of which has been observed. Finally, adventitial cystic disease may be part of a systemic degenerative disease. However, there are no reported cases of contralateral lesions developing or of a second lesion at another location.

The diagnosis of adventitial cystic disease should be suspected in a young nonsmoker with sudden onset of claudication. As noted above, physical findings can include a popliteal bruit, loss of pulses with knee flexion, or absent distal pulses. Angiography may show a characteristic "scimitar sign," or smooth, eccentric narrowing representing luminal encroachment by the cyst. Less invasive diagnostic techniques have been successful in establishing the diagnosis, including ultrasound and MRI. ${ }^{24}$

Treatment of adventitial cystic disease has been highly successful. In Flanigan's review, treatment was fully successful in about $90 \%$ of cases. ${ }^{23}$ In only one case out of 115 did treatment failure lead to amputation. The authors 
advocate cyst excision when the arterial lumen is intact, and interposition grafting if the artery is thrombosed or extensively replaced by cysts. This approach has been borne out in a review of more recent experience as well. ${ }^{22}$ Needle aspiration of cyst fluid, either percutaneously under image guidance or during open surgery, has also been used. Results of this approach have been variable, with recurrence rates of approximately $10 \%$. In one report, however, seven patients were treated with ultrasound guided percutaneous aspiration with no recurrences at 1- to 32-month follow-up. These authors suggest that this technique may be the treatment of choice. ${ }^{25}$

\section{EXERCISE INDUCED COMPARTMENT SYNDROME}

Exercise induced compartment syndrome can cause calf pain clinically identical to claudication with vigorous activity. Sports activities lead to elevated muscle volume that results in elevated comparment pressures if fascial compartments are excessively tight. ${ }^{26}$ In one study of patients with a clinical diagnosis of exercise-induced calf compartment syndrome, symptomatic patients were compared with controls to assess the effect of exercise on compartment pressures. Both groups had similar resting pressures of approximately $\mathbf{5}$ $\mathrm{mmHg}$. In the symptomatic patients, pressures rose with exercise to nearly $20 \mathrm{mmHg}$, whereas in the controls, pressures fell by half. After fasciotomy, the normal pressure drop with exercise was restored, and pain was relieved. ${ }^{27}$

The diagnosis of chronic compartment syndrome depends on clinical suspicion and measurement of compartment pressures at rest and with exercise. In fact, it may be necessary for the patient to perform the specific exercise that induces the symptoms to elicit the elevated pressures. ${ }^{28}$ Doppler pressures and pulse-volume recordings are expected to be normal. ${ }^{4}$ Treatment requires release of the involved fascial compartment by fasciotomy or fasciectomy.

\section{REPETITIVE TRAUMA}

Highly trained athletes have been reported to develop arterial lesions caused by repetitive trauma. Dissection of the external iliac artery has been reported in endurance athletes between 40 and 50 years of age. The presentation is one of acute onset of short distance claudication associated with vigorous endurance training or competition. The pathologic lesion is isolated dissection of the external iliac artery with no other arterial abnormality. Treatment with reconstruction or interventional techniques has been successful. ${ }^{29}$

Similarly, the external iliac artery can be affected by a stenotic intimal thickening caused by progressive fibrosis. This lesion has been reported almost exclusively in high-level competition cyclists. Presumably repetitive, strenuous hip flexion results in a particular pattern of arterial trauma that causes this unique lesion. The presentation is of thigh claudication during 
maximal strain while cycling. Patients have been managed with arterial reconstruction which allows many patients to return to competition. ${ }^{30,31}$

\section{CONGENITAL ANOMALIES}

Claudication in young adults can, on rare occasions, be a late presentation of a congenital arterial anomaly. Aortic coarctation typically presents with severe hypertension early in life, and in 98\% of cases the lesion is located in the aortic arch at the level of the ligamentum arteriosum. ${ }^{32-34}$ Those cases in which the abdominal aorta is the site of coarctation generally present with renovascular hypertension, either because the stenosis is above the level of the renal arteries or because of separate stenosis of the renal arteries themselves. In these cases, claudication is a secondary consideration. On the other hand, if the lesion is located infrarenally and the renal arteries are spared, symptoms may be limited to claudication. These patients tend to present somewhat later, in early adulthood. ${ }^{35}$ Patients with new onset claudication presenting in the fifth decade have also been reported. However, these patients may have an acquired form of coarctation from an inflammatory etiology. ${ }^{36}$

Treatment of abdominal aortic coarctation has generally consisted of thoracic aorta-to-infrarenal aorta bypass using prosthetic graft. In cases of renovascular hypertension caused by separate renal artery stenosis, renal revascularization is generally accomplished through aortorenal bypass originating from the graft. Alternate methods of reconstruction that have been reported include prosthetic patch aortoplasty, bypass using the splenic artery, or bypass using homograft. ${ }^{34-36}$

Another congenital anomaly that has been implicated in claudication is the persistent sciatic artery. During embryologic development, the sciatic artery supplies the limb bud of the lower extremity. As development proceeds, this artery involutes and the arterial supply to the leg is taken over by the external iliac and femoral arteries. If this process of involution fails, the sciatic artery can persist into adulthood as an extension of the hypogastric artery through the buttock, into the thigh, and in cases of complete persistence, continuous with the popliteal artery. ${ }^{37}$ The persistent sciatic artery can coexist with a relatively normal femoral artery, or the femoral artery can be hypoplastic or absent.

About $40 \%$ of persistent sciatic arteries will develop aneurysms, which can be detected as a pulsatile mass in the buttock. If these aneurysms thrombose or embolize, the result can be limb threatening ischemia or claudication. ${ }^{37,38}$ Furthermore, patients may develop claudication in the absence of aneurysm formation if the abnormal artery is insufficiently developed to supply the limb with normal blood flow. ${ }^{39}$ In the absence of aneurysm, treatment is with femoropopliteal bypass. If an aneurysm is present, treatment requires ligation of the aneurysm via a posterior approach, with femoropopliteal bypass if the femoral artery is absent or hypoplastic. 


\section{ERGOTISM}

Historically, toxicity from ergot alkaloids was caused by ingestion of grain contaminated by the fungus Claviceps purpurea. The resulting vasospasm caused ischemic neuropathy and a burning pain known as St. Anthony's fire ${ }^{40}$ Modern ergotism is caused by use or abuse of ergotamine-containing drugs used for the treatment of migraine headaches. Several reports describe patients complaining of cool, cyanotic extremities and intermittent claudication. ${ }^{40-42}$ The mechanism of the vasospasm is the direct action of ergotamine on smooth muscle that occurs at high doses.

The first line of treatment for ergotism is discontinuation of the drug. Vasospasm typically resolves within a few days. Several vasodilating agents have been used in an attempt to reverse the vasospasm, but have had limited success. Tolazoline and sodium nicotinate are the only agents that have been shown to have an immediate clinical benefit, and these results are limited to case reports. ${ }^{42}$ Because severe vasospasm has been associated with arterial thrombosis, anticoagulation with heparin or dextran may be indicated in cases of impending gangrene. ${ }^{40}$

Despite the fact that ergotamine-induced ischemia represents a toxic effect of the medication, claudication and persistent ischemia can persist chronically, even after discontinuation of the drug. In one report, a patient presented with 7 months of claudication caused by ergotamine and continued to suffer symptoms one year after stopping the medication. ${ }^{41}$ Angiogram revealed persistent stenosis of the external iliac arteries, which in the face of continued symptoms, was treated by atherectomy. A focal stenosis in this setting may be amenable to angioplasty, but cases of ergotism that require arterial intervention are rare, and no conclusions can be drawn as to the best method of reconstruction.

\section{SUMMARY}

Claudication in young patients has important differences from the more familiar clinical entity in the older age group. The clinician faces greater challenges in making the diagnosis of a vascular cause of claudication, which is generally unexpected in the young. These patients typically have higher expectations and needs in terms of functional results. Crucial to effective management of these patients is a clear understanding of the distinct spectrum of pathophysiology that causes claudication in the young age group.

\section{REFERENCES}

1. Levy PJ, Hornung CA, Haynes JL, et al. Lower extremity ischemia in adults younger than forty years of age: a community-wide survey of premature atherosclerotic arterial disease. J Vasc Surg 1994;19:873-881

2. Sise MJ, Shackford SR, Rowley WR, et al. Claudication in young adults: a frequently delayed diagnosis. J Vasc Surg 1989;10:68-74 
3. Jelnes R, Gaardsting $\mathrm{O}$, Jensen $\mathrm{KH}$, et al. Fate in intermittent claudication: outcome and risk factors. Br Med J 1986;293:1137-1140

4. Porter JM, Taylor LM, Harris EJ. Nonatherosclerotic vascular disease. In: Moore WS, ed. Vascular surgery, a comprehensive review. Philadelphia: WB Saunders; 1993:108-145

5. McCready RA, Vincent AE, Schwartz RW, et al. Atherosclerosis in the young: a virulent disease. Surgery 1984;96:863-868

6. Bouhoutsos J, Martin P. The influence of age on prognosis after arterial surgery for atherosclerosis of the lower limb. Surgery 1973;74:637-640

7. Valentine RJ, MacGillavray DC, DeNobile JW, et al. Intermittent claudication caused by atherosclerosis in patients aged forty years and younger. Surgery 1990;107:560-565

8. De Bakey ME, Crawford ES, Garrett HE, et al. Occlusive disease of the lower extremities in patients 16 to 37 years of age. Ann Surg 1964;159:873-890

9. Levy PJ, Gonzales MF, Hornung CA, et al. A prospective evaluation of atherosclerotic risk factors and hypercoagulability in young adults with premature lower extremity atherosclerosis. J Vasc Surg 1996;23:36-45

10. Eldrup-Jorgensen J, Flanigan DP, Brace L, et al. Hypercoagulable states and lower limb ischemia in young adults. J Vasc Surg 1989;9:334-341

11. DeLaurentis DA, Friedman P, Wolferth CC, et al. Atherosclerosis and the hypoplastic aortoiliac system. Surgery 1978;83:27-37

12. Levy PJ, Tabares AH, Olin JW. Lower extremity arterial occlusions in young patients with Crohn's colitis and premature atherosclerosis: report of six cases. Am J Gastroenterol 1997;92:494-497

13. VanGoor H, Boontje AH. Results of vascular reconstructions for atherosclerotic occlusive disease of the lower limbs in young adults. Eur J Vasc Endovasc Surg 1995;10:323-326

14. Gibson MHL, Mills JG, Johnson GE, et al. Popliteal entrapment syndrome. Ann Surg 1977; 185:341-348

15. Persky JM, Kempczinski RF, Fowl RJ. Entrapment of the popliteal artery. Surg Gynecol Obstet 1991;173:84-90

16. Rich NM, Collins GJ, McDonald PT. Popliteal vascular entrapment. Arch Surg 1979; 114:1377-1384

17. Turnipseed WD, Pozniak M. Popliteal entrapment as a result of neurovascular compression by the soleus and plantaris muscles. J Vasc Surg 1992;15:285-294

18. Fowl RJ, Kempczinski RF. Popliteal artery entrapment. In: Rutherford RB ed. Vascular Surgery. Philadelphia: WB Saunders; 2000:1087-1093

19. Di Marzo L, Cavallaro A, Sciacca V, et al. Diagnosis of popliteal artery entrapment syndrome: the role of duplex scanning. J Vasc Surg 1991;13:434-438

20. Erdoes LS, Devine JJ, Bernhard VM, et al. Popliteal vascular compression in a normal population. J Vasc Surg 1994;20:978-986

21. Lambert AW, Wilkins DC. Popliteal artery entrapment syndrome: collaborative experience of the Joint Vascular Research Group. Br J Surg 1998;85:1367-1368

22. Levien LJ, Benn CA. Adventitial cystic disease: a unifying hypothesis. J Vasc Surg 1998; 28:193-205

23. Flanigan DP, Burnham SJ, Goodreau JJ, et al. Summary of cases of adventitial cystic disease of the popliteal artery. Ann Surg 1979;189:165-175

24. Miller A, Salenius JP, Sacks BA, et al. Noninvasive vascular imaging in the diagnosis and treatment of adventitial cystic disease of the popliteal artery. J Vasc Surg 1997;26: 715-720

25. Do DD, Braunschweig M, Baumgartner I, et al. Adventitial cystic disease of the popliteal artery: percutaneous US-guided aspiration. Radiology 1997;203:743-746

26. Martens MA, Moeyersoons JP. Acute and recurrent effort-related compartment syndrome in sports. Sports Med 1990;9:62-68 
27. Biedert RM, Marti B. Intracompartmental pressure before and after fasciotomy in runners with chronic deep posterior compartment syndrome. Int J Sports Med 1997;18:381-386

28. Padhiar N, King JB. Exercise induced leg pain-chronic compartment syndrome. Is the increase in intra-compartment pressure exercise specific? Br J Sports Med 1996;30: 360-362

29. Cook PS, Erdoes LS, Selzer PM, et al. Dissection of the external iliac artery in highly trained athletes. J Vasc Surg 1995;22:173-177

30. Abraham P, Chevalier JM, Leftheriotis G, et al. Lower extremity arterial disease in sports. Am J Sports Med 1997;25:581-584

31. Rousselet MC, Saint-Andre JP, L'Hoste P, et al. Stenotic intimal thickening of the external iliac artery in competition cyclists. Hum Pathol 1990;21:524-529

32. Shanley CJ, Stanley JC. Coarctation and hypoplasia of the subisthmic thoracic and abdominal aorta. In: Ernst CB, Stanley JC eds. Current Therapy in Vascular Surgery. St Louis: Mosby; 1995:359-363

33. Hallett JW, Brewster DC, Darling RC, et al. Coarctation of the abdominal aorta: current options in surgical management. Ann Surg 1980;191:430-437

34. Scott HW, Dean RH, Boerth R, et al. Coarctation of the abdominal aorta: long-term results of surgical therapy. Ann Surg 1979;189:746-755

35. Mickley V, Fleiter T. Coarctations of the descending thoracic and abdominal aorta: current options in surgical management. J Vasc Surg 1998;28:206-214

36. Sproul G, Pinto J. Coarctation of the abdominal aorta. Arch Surg 1972;105:571-573

37. McLellan GL, Morettin LB. Persistent sciatic artery: clinical, surgical, and angiographic aspects. Arch Surg 1982;117:817-822

38. Brantley SK, Rigdon EE, Raju S. Persistent sciatic artery: embryology, pathlogy, and treatment. J Vasc Surg 1993;18:242-248

39. Bower EB, Smullens SN, Parke WW. Clinical aspects of persistent sciatic artery: report of two cases and review of the literature. Surgery 1977;81:588-595

40. Greene FL, Ariyan S, Stansel HC. Mesenteric and peripheral vascular ischemia secondary to ergotism. Surgery 1977;81:176-179

41. Braunlich S, Kroger K, Massalha K, et al. Ergotamine-induced intermittent claudication. Vasa 1999;28:123-126

42. Merhoff GC, Porter JM. Ergot intoxication: historical review and description of unusual clinical manifestations. Ann Surg 1974;180:773-779 
Drs. Krug, Calligaro, and Dougherty have outlined the current information available on the young patient with claudication. My comments will add only my personal perspective on the incidence and treatment of this problem. This is timely because my perception is that we are all seeing a larger number of these patients. They are challenging and their optimal treatment depends on understanding differences in the causes and natural history of claudication in the 40-year-old as compared to the 65-year-old.

What is surprising about this problem is how little is known about the true incidence of claudication in young patients. Perhaps the best evidence we have is from the Framingham Study where 2.3\% of men age 35-44 years reported symptoms consistent with claudication. ${ }^{1}$ Data from retrospective practicebased surveys are important and useful but have limitations. First of all, these studies rarely identify young patients with mild symptoms. The diagnosis becomes nearly inescapable to the primary care giver when advanced clinical signs of ischemia occur. This leads to referral to a vascular laboratory. Thus, the most severe cases always come to attention, while milder disease remains undiagnosed. Population-based screening studies are difficult to interpret because most are based on questionnaires. Since we really don't know the denominator, it is difficult to substantiate our belief that patients who develop vascular disease at an earlier age have more rapid progression and a poor outcome. This conclusion is probably biased by the select group of young patients with more severe disease that reach our offices.

The high incidence of hyercoagulability in this group adds to the challenge of providing their care. This raises an important practical question regarding the cost of evaluation for hypercoaguable states. Thrombocytosis and connective tissue diseases are treated by specific therapies that require accurate diagnosis. The remainder of the hypercoagulable states are treated with longterm coumadin anticoagulation. Is it of practical value to identify specific coagulation defects? My current approach is to perform cell counts for thrombocytosis and serologic studies for connective tissue disorders. If these are negative, I empirically treat these patients with life-long coumadin after intervention. Obviously, individual considerations such as an occupation associated with a high risk of trauma, alcoholism, or unreliability have to be taken in account when considering long-term anticoagulation.

If claudication is caused by atherosclerosis in a young person, then we need to look for treatable factors contributing to accelerated atherosclerosis.

B.T.B., Vascular Surgeon, Department of Surgery, Division of Vascular Surgery; University of Nebraska Medical Center, Omaha, NE.

Copyright (c) 2000 by Thieme Medical Publishers, Inc., 333 Seventh Avenue, New York, NY 10001, USA.

Tel.: +1(212) 584-4662. 0894-8046,p;2000,13,2,39,40,ftx,en; pvs00099A 
Tobacco use is usually the single most important contributing factor. I insist that the patient enroll in, and complete, a smoking cessation program before performing any elective intervention. Hypercholesterolemia can be effectively treated. Identification of hyperhomocysteinemia is important because it is treatable. Additionally, there is a growing body of evidence to suggest that active inflammation may be important in progression of atherosclerosis. ${ }^{2}$ If Creactive protein levels are high, I add aspirin to the coumadin regimen. This is not associated with an excessive risk of bleeding in this patient population.

These patients are challenging. They require multi-modality therapy that combines standard revascularization techniques with aggressive treatment of their hypercoagulable, serologic, metabolic, and systemic inflammatory states. The group from Pennsylvania Hospital has done a very nice job of laying out the background for our current approach to these patients.

\section{REFERENCES}

1. Kannel WB, Shurleff D. The Framingham study. Cigarettes and the development of intermittent claudication. Geriatrics 1973;28:61

2. Ridker PM, Cushman M, Stampfer JJ, et al. Inflammation, aspirin, and the risk of cardiovascular disease in apparently healthy men. N Engl J Med 1997;336:973-979 
We appreciate Dr. Baxter's comments and agree with his points concerning claudication in young patients. Although population based screening studied may be flawed, and a select group of young patients with more advanced disease are referred to vascular surgeons, most series report lower patency and limb-salvage rates in patients younger than 50 years old compared to older patients. Although cost-effectiveness of hypercoaguable screening in these patients prior to performing a lower extremity revascularization is difficult to prove, we generally obtain hypercoaguable laboratory studies preoperatively and frequently will administer life-long anticoagulation for lower extremity bypasses in patients younger than 50 years old. We also agree with Dr. Baxter's recommendations concerning identifying risk factors for atherosclerosis and trying to modify them prior to intervention. 
